\title{
INOVAÇÃO NO CONTEXTO DE CONVERGÊNCIA TECNOLÓGICA EM BIOTECNOLOGIA:UM ESTUDO DE CASO
}

Victor Prochnik Instituto de Economia da UFRJ

Jorge Britto

Departamento de Economia da UFF

Ricardo L. Torres

Departamento Acadêmico de Gestão e Economia da UTFPr

\author{
Lia Hasenclever \\ Instituto de Economia da UFRJ
}

\begin{abstract}
RESUMO
O artigo aborda a difusão da biotecnologia na indústria farmacêutica brasileira, discutindo o caso de uma empresa de capital brasileiro. A diversificação tecnológica na direção de uma tecnologia genérica como a biotecnologia caracteriza a inovação por convergência tecnológica (CT). Busca-se compreender especificidades de processos de CT e seus impactos sobre as trajetórias de crescimento, do ponto de vista do interesse na proposição de políticas tecnológicas e industriais. Em particular, o estudo de caso mostra a importância das relações entre processos de CT, por um lado, e de estratégias empresariais de recursos humanos para a inovação, por outro. Essas relações são mais complexas quando há o recurso a modelos de inovação aberta, em geral usados em processos de CT.
\end{abstract}

\begin{abstract}
The paper addresses the biotechnology diffusion in the Brazilian pharmaceutical industry, discussing the case of a Brazilian-owned firm. The technological diversification in the direction of a general purpose technology as biotechnology characterizes the innovation in technological convergence (TC). It is sought to understand the specificities of TC processes and their impacts on the growth trajectory of the firm, from the viewpoint of technology policies propositions. In particular, the case study shows the existence of relations between TC processes, on the one hand, and human resource strategies for innovation, on the other hand. These relations are more complex under the open innovation paradigm, generally followed in TC processes.
\end{abstract}

PALAVRAS CHAVE: convergência tecnológica, pesquisa e desenvolvimento, inovação aberta, política tecnológica

KEY WORDS: technological innovation, research and development, open innovation, technology policy.

CLASSIFICAÇÃO JEL: 031 
O artigo aborda a difusão da biotecnologia na indústria farmacêutica brasileira, apresentando o caso de uma das empresas líderes neste processo, o Laboratório Cristália. A biotecnologia é uma tecnologia genérica de grande impacto intersetorial e está na base de um amplo processo de convergência tecnológica. No estudo da diversificação tecnológica para a biotecnologia, busca-se, em geral, compreender especificidades de processos de inovação em CT e seus requisitos. . Em particular, o estudo de caso mostra a importância das relações entre processos de CT, por um lado, e de estratégias empresariais de recursos humanos para a inovação, por outro. Essas relações são mais complexas quando há o recurso a modelos de inovação aberta, em geral usados em processos de CT.

Na discussão do processo de convergência tecnológica, optou-se por centrar o foco no campo biotecnológico. A utilização da moderna biotecnologia para a obtenção de novos bens, processos e serviços envolve uma combinação de múltiplos blocos de conhecimento e tecnologias, cujas aplicações transversais vêm impactando a heurística de solução de problemas e busca por inovações em diversos setores, como agropecuária e as indústrias de alimentos, química e farmacêutica (FONSECA, 2009; NETO et al, 2009). Mas os desafios técnicos para uma entrada bem-sucedida de uma empresa de um país emergente como o Brasil na produção de biológicos não são triviais. Ao enfatizar o estudo de variáveis de recursos humanos no estudo destes desafios, coloca-se, como questão metodológica importante, o nível de recorte analítico mais adequado para a compreensão do fenômeno. Neste sentido, observa-se que no Brasil, assim como em outros países em desenvolvimento, a investigação empírica e a literatura sobre o papel dos recursos humanos na inovação em CT é muito escassa, carecendo de evidência mais detalhadas captadas no plano microeconômico. De modo a contribuir para diminuir essa carência de informações, optou-se elaborar a análise a partir de um estudo de caso, capaz de levantar questões práticas e hipóteses teóricas e indicar caminhos a seguir, para a pesquisa futura em economia da inovação.

O artigo estrutura-se em quatro seções. Inicialmente, procura-se problematizar a noção de Convergência Tecnológica (CT), referenciando essa análise às características do processo de P\&D no plano empresarial e às estratégias internas e externas de desenvolvimento tecnológico. A segunda seção discute a trajetória da recente biotecnologia como evidência de um processo de Convergência Tecnológica com impactos diretos sobre o setor farmacêutico, procurando associar essa discussão à experiência brasileira recente de fortalecimento das capacitações inovativas de empresas de capital nacional no setor. A terceira seção mostra a metodologia seguida e a quarta apresenta o caso do Laboratório Cristália, procurando avaliar como o impacto da biotecnologia sobre a dinâmica tecnológica e competitiva do setor de atuação da empresa resultou em estímulos a processos de diversificação tecnológica e ao fortalecimento das suas capacitações inovativas, segundo um padrão de "inovação aberta" alinhado com os desafios enfrentados pela empresa.

\section{CONVERGÊNCIA TECNOLÓGICA E ESTRATÉGIAS DE DESENVOLVIMENTO TECNOLÓGICO}

Nesta seção, procura-se 1) apresentar o conceito de CT, 2) mostrar a crescente relevância da inovação em CT, 3) indicar o forte impacto esperado deste tipo de inovação sobre o desenvolvimento econômico e 4) detalhar duas características da inovação em CT, a política diferencial para os recursos humanos e a ênfase na inovação aberta..

\subsection{A relevância da inovação em CT}

Para Rosenberg (1963), que cunhou o conceito, a CT é a crescente semelhança entre bases técnicas para fabricar produtos que diferem quanto ao seu uso. Ele aplicou o conceito à evolução da indústria de máquinas ferramentas, no século XIX, mostrando que as bases técnicas das empresas deste setor e, 
também, das empresas clientes se aproximavam, por causa da difusão de inovações. Por exemplo, um sistema de lubrificação criado por uma empresa da indústria de bicicletas passou a ser usado na indústria de máquinas ferramentas e, mais tarde, foi transferido para a indústria automobilística.

O conceito vem evoluindo. Artigos recentes se referem à CT como "o processo de tornar indistintas (blurring) as fronteiras entre duas ou mais indústrias diferentes ao combinar seu conhecimento científico, tecnologias e mercados” - (PRAHALAD; RAMASWAMY, 2003) e (KIM et al., 2015). Ela pode ocorrer pela integração entre duas ou mais tecnologias (na evolução do telefone celular, por exemplo) ou pela substituição de uma tecnologia por outra (visível nos aparelhos de som).

Kim et al. (2015) analisam processos de convergência em todos os setores da economia dos Estados Unidos, procurando por palavras chaves em cerca de dois milhões de artigos de jornal, entre 1989 e 2012. Os dados indicam um continuado e expressivo aumento no número de processos de CT no tempo.

Mas a visão de CT como processo de blurring entre indústrias não permite diferenciar a convergência tecnológica de estratégias pontuais de diversificação tecnológica. A convergência tecnológica se refere, mais propriamente, aos casos de adoção das chamadas tecnologias genéricas (GAMBARDELLA; TORRISI, 1998, p. 446) ou tecnologias de propósito geral (GPTs, general purpose technologies) (CANTNER; VANNUCCINI, 2014). Estas tecnologias, com ampla difusão pela economia, aproximam a base de conhecimento tecnológico dos processos de produção de vários setores, anteriormente diferentes entre si.

Kim et al (2015) também mostram que não há uma homogeneidade entre os processos de CT e alguns tendem à maturidade e outros à expansão, como visto a seguir. Um exemplo de processo de convergência amplamente conhecido é o das tecnologias de informação e comunicação. Basole et al $(2014,1)$ procuraram "quantificar e visualizar a dinâmica competitiva que forma o ecossistema de tecnologias da informação e comunicação.”. Eles concluem que “a taxa de convergência está diminuindo. Isto sugere a crescente maturidade do ecossistema e menor importância das relações formais entre firmas.” (BASOLE; PARK; BARNETT, 2014, 1). Outro exemplo é o da bioeconomia, que envolve a agricultura, com destaque para a bio-energia, indústria farmacêutica, indústria de alimentos, química orgânica, etc. (GOLEMBIEWSKI; SICK; BRÖRING, 2015) concluíram que o processo de CT em bioeconomia está em fase inicial, predominantemente de criação de conhecimento científico e tecnológico.

Espera-se não apenas a continuidade dos processos de convergência em tecnologias digitais e bioeconomia como a sua intercessão, o que deve vir a ter um papel central no desenvolvimento econômico e tecnológico nas próximas décadas. Já existem políticas industriais e tecnológicas voltadas para acelerar a evolução, fusão e a difusão das tecnologias genéricas. Segundo (DRATH; HORCH, 2014) e (SCHWAB, 2016), estamos no limiar da Quarta Revolução Industrial (4RI). A 4RI é entendida como a era da aplicação dos Cyber-physical systems (CPS) aos sistemas de produção. Cyber-phisical systems “ are engineered systems that are built from, and depend upon, the seamless integration of computational algorithms and physical components” (NATIONAL SCIENCE FOUNDATION, 2014, 2). A 4RI é o resultado das confluências entre as tecnologias da informação e comunicação, inteligência artificial, biotecnologia, ciência dos materiais entre outras. "Many of these innovations are in their infancy, but they are already reaching an inflection point in their development as they build on and amplify each other in a fusion of technologies across the physical, digital and biological worlds.” - (SCHWAB, 2016, 1)

Neste contexto internacional, cabe perguntar sobre as oportunidades e desafios para a indústria brasileira. A dificuldade de constituir um sistema técnico-econômico de geração e consumo da tecnologia é um obstáculo para a internalização ativa da tecnologia de propósito genérico na economia. Este artigo analisa o esforço de uma empresa em incorporar a nova tecnologia genérica e, em particular, absorver conhecimento no contexto do ainda fraco desenvolvimento deste sistema técnico-econômico no Brasil. Em particular, ele mostra que estudos nesta área devem enfatizar a atuação das políticas de recursos 
humanos da firma que são ainda mais importantes em um contexto de estratégias de inovação aberta. Como visto adiante, a ênfase na análise da política de RH destoa da grande maioria dos estudos sobre inovação no Brasil, que não consideram esta dimensão do problema. A crescente adoção do paradigma da inovação aberta amplia a relevância das estratégias de recursos humanos para inovação, abrindo novas questões para a política industrial e tecnológica.

\subsection{Duas características do processo de P\&D em convergência tecnológica}

Em uma empresa, o processo de inovação em CT se dá através do desenvolvimento/ introdução de novas tecnologias já usadas em outros setores, mas desconhecidas para a firma. Um processo de CT significativo se constitui em um salto qualitativo na evolução da firma, por requerer uma descontinuidade técnica. Ele modifica a base tecnológica com que a firmas opera. Os seus impactos, possivelmente, atingem todas as demais áreas da empresa e, também, suas conexões externas.

As atividades internas de $P \& D$ sempre foram vistas como a alavanca mais importante do desenvolvimento tecnológico. Também, o gasto em P\&D interno está relacionado ao externo, na medida em que reforça a capacidade de absorção de novo conhecimento. Mas, nas inovações em CT, essas relações não são válidas, pois é requerido conhecimento novo, em geral pouco associado ao conhecimento detido pela firma. As empresas, portanto, precisam se qualificar para introduzir novas tecnologias.

Duas opções complementares para a qualificação são a capacitação e ampliação do pessoal interno e o acesso a novas tecnologias, recorrendo a fontes de informações externas ou à cooperação com outros agentes econômicos, vistos a seguir.

\subsubsection{Estratégias internas de desenvolvimento tecnológico}

A análise do RH é valorizada em teorias estratégicas da firma que enfatizam fatores internos às empresas como fontes de vantagens competitivas. Este é o caso, por exemplo, da RBV (resource based view) (BARNEY; WRIGHT, 1997), perspectiva das capacitações dinâmicas (TEECE et al., 1997) e da teoria evolucionista ou neo-schumpeteriana (NELSON; WINTER, 2009). Nestas escolas, conceitos importantes para a análise da vantagem competitiva, como aprendizado, conhecimento, capacitações dinâmicas etc. se baseiam, essencialmente, na capacidade, atuação e interação das pessoas e equipes. - (WRIGHT; DUNFORD; SNELL, 2001).

Entre as pessoas ocupadas, as que trabalham em P\&D têm importância central nestas teorias, pela sua contribuição para a constituição das capacitações dinâmicas, vantagens competitivas etc.. Assim, a inclusão de variáveis relativas ao pessoal empregado em $\mathrm{P} \& \mathrm{D}$, em particular sua composição, é relevante em estudos em inovação, principalmente no caso da inovação em CT.

Em paralelo, a resenha de Zanello et all (2015), sobre a inovação nos países em desenvolvimento, indica, enfaticamente, artigos que mostram a importância dos recursos humanos para a inovação (laços sociais dos gestores, empreendedorismo etc. por exemplo). Os autores afirmam "Recurrently we also find in the literature references to factors internal to the firm that can stand in the way of innovation and affect the competitive strategy of a firm. The predominant factors are the lack of human capital (education and managerial skills), resources (financial capital and information) and networking capabilities.” (ZANELLO et al., 2015, 15). Eles não mencionam artigos sobre o pessoal em P\&D, o que pode estar mostrando que esta é uma lacuna importante na literatura.

A CT requer a capacitação da equipe de P\&D nas novas tecnologias. Mas as possibilidades de atualização do pessoal existente são, em geral, limitadas pelo tempo disponível e pela extensão das novas áreas de conhecimento requeridas. A alternativa é a contratação de novos profissionais, especializados nas tecnologias que estão sendo introduzidas. Assim, a inovação em CT leva, em geral, à ampliação do leque 
de capacidades e, também, de profissões na empresa. O aumento da variedade na composição da formação da mão de obra em P\&D é um aspecto relevante do esforço interno às firmas no sentido da inovação CT.

Mas são escassos os estudos sobre a composição e a evolução do pessoal em P\&D no Brasil. Até 2008, a pesquisa da inovação tecnológica brasileira, PINTEC/IBGE, perguntava sobre o número de pessoas em P\&D por ocupação profissional, agrupadas em seis categorias: Químicos, físicos e assemelhados, 2) Engenheiros, arquitetos e assemelhados, 3) Médicos, cirurgiões dentistas, veterinários, enfermeiros e assemelhados, 4) Biologistas, bacteriologistas, farmacologistas e assemelhados e 5) Estatísticos, matemáticos, analistas de sistemas e 6) outros. Estas perguntas foram posteriormente retiradas do questionário da PINTEC, por terem sido muito pouco utilizadas.

Uma pesquisa bibliográfica não encontrou nenhum estudo econométrico usando estas variáveis. O único estudo descritivo, (MAZZONI et al, 2013), mostra haver uma grande concentração de engenheiros, o que pode explicar o baixo apelo das perguntas. Na indústria de transformação, entre as 21.225 pessoas em atividades de P\&D em 2003, 74,2\% eram engenheiros. Mas este percentual caiu para 66,4\% em 2008, um indicador de que houve uma dispersão na composição da mão de obra em P\&D. No setor químico, que abrange a indústria farmacêutica, os respectivos percentuais são 35,2\% e 22,7\%.

Há outros estudos, embora poucos, sobre mão de obra em $P \& D$, usando as demais variáveis encontradas na PINTEC e, também, utilizando respostas de pesquisas por questionário etc. Ver, por exemplo, (TEIXEIRA, 2016).

Espera-se, portanto, que os resultados deste estudo de caso incentivem a pesquisa e a discussão sobre a relação entre a estratégia de $\mathrm{RH}$ em $P \& D$ e o desempenho inovativo da firma, diminuindo o uso de variáveis proxy como o gasto em $\mathrm{P} \& \mathrm{D}$, em inovação etc. O banco de dados da RAIS, entre outras fontes, pode ser útil para este fim.

\subsubsection{Estratégias extermas de desenvolvimento tecnológico}

Uma alternativa para desenvolver inovações em CT é o recurso a conhecimento externo à firma, possivelmente em complementaridade com o investimento em $\mathrm{RH}$ internos. As informações técnicas podem ter, como origem, outros setores da empresa (departamentos de P\&D em outros países, por exemplo), agentes externos ou outros locais em que elas são disponíveis.

Essa prática é estudada no conceito de inovação aberta: "the use of purposive inflows and outflows of knowledge to accelerate internal innovation, and expand the markets for external use of innovation, respectively.” - (CHESBROUGH, 2006)

A inovação aberta substancialmente modifica as atividades das pessoas ocupadas em P\&D previstas nas vertentes teóricas mencionadas (visão baseada em recursos e teoria das capacitações dinâmicas). Por exemplo, para a resource based view, recursos internos levam à vantagem competitiva quando são valiosos, raros, difíceis de serem imitados e coerentes com a estrutura da organização (BARNEY, 1991). Essas vertentes colocam o foco da análise na criação de sinergias internas e na coerência interna das organizações.

Mas o paradigma da inovação aberta é o de que existe conhecimento externo crucial para a inovação. No paradigma da inovação aberta, as competências são criadas a partir da interação das atividades internas e externas de P\&D. Esta complementaridade precisa ser construída. Para isto, são requeridas fortes modificações na organização dos departamentos de P\&D e nos tipos de rotinas realizadas pelas pessoas. O modelo de inovação aberta privilegia a descentralização e o compartilhamento de informações, o desenvolvimento de parcerias, a coordenação de projetos diferentes feitos por agentes diversos, cultura de 
cooperação etc. A gestão da integração é agora uma tarefa fundamental. Essas mudanças é que tornam as atividades internas de P\&D mais complexas e requerem mais da força de trabalho interna da organização, isto é, valorizam a importância da política de RH na inovação.

\section{METODOLOGIA}

O caso estudado contempla uma empresa de capital brasileiro do setor farmacêutico, originariamente especializada no segmento químico-farmacêutico e em processo de capacitação e diversificação tecnológica para a moderna biotecnologia. Não foi escolhida uma subsidiária de empresa multinacional porque essas empresas têm a vantagem de contar com o apoio da matriz e de outras subsidiárias do mesmo grupo econômico onde, em geral, o processo de CT ocorreu anteriormente.

O foco da análise direciona-se para o departamento de $\mathrm{P} \& \mathrm{D}$, perguntando-se por mudanças que ocorreram, obstáculos encontrados e insucessos e sucessos alcançados. Estas perguntas encontram-se associadas às duas questões de interesse central, a ampliação das capacitações em P\&D e o recurso à inovação aberta para alcançar convergência tecnológica.

Este trabalho foi feito a partir de uma pesquisa maior que analisou respostas de sete laboratórios farmacêuticos privados de capital brasileiro. Entre elas, foi selecionada a mais atuante em inovação, coincidentemente a que mais avançou na direção da biotecnologia. A firma selecionada é um dos laboratórios privados de controle brasileiro líderes na introdução da biotecnologia.

Na empresa selecionada, o pesquisador passou quatro dias (novembro/ 2014). Além de visitar as fábricas e conversar com outras pessoas, ele realizou 13 entrevistas, previamente estruturadas, na área de desenvolvimento organizacional $(\mathrm{RH})$, coordenação de projetos, P\&D (2), diretoria industrial (2), diretoria de biotecnologia, diretoria de farmoquímica, patentes, logística, assuntos regulatórios, diretoria de qualidade e diretoria comercial.

A empresa também respondeu um questionário sobre informações específicas, como receita, gastos, número de pessoas, patentes etc. O questionário foi validado por três profissionais da indústria e suas sugestões deram origem a uma segunda versão. Posteriormente, houve três entrevistas piloto, que também possibilitaram melhorar o questionário.

\section{IMPACTOS DA CONVERGÊNCIA EM BIOTECNOLOGIA SOBRE O SETOR FARMACÊUTICO BRASILEIRO}

\subsection{Tendências Gerais e Desafios para o Brasil}

$\mathrm{Na}$ indústria farmacêutica, a crescente incorporação da biotecnologia como base de conhecimento e ferramenta para o desenvolvimento de inovações evidencia um processo de convergência tecnológica que aproxima e integra conhecimentos tradicionais no campo da síntese química com esforços orientados à manipulação de processos biológicos e organismos vivos em nível genético e molecular.

Estima-se que, em 2017, os medicamentos biológicos venham a representar cerca de 20\% (US\$ 220 bilhões) do mercado de medicamentos (IMS Health). Esses medicamentos são os que têm a maior taxa de crescimento. Entre 2010 e 2015 o crescimento médio anual do mercado mundial desses produtos foi de 
9,9\% ao ano. Até 2018, sete dentre os dez produtos farmacêuticos mais vendidos no mundo serão medicamentos biotecnológicos (Evaluate Pharma, 2013). No Brasil, entre 2005 e 2010, as importações de produtos biológicos aumentaram, em média, 37\% ao ano, com oito entre os 10 principais medicamentos com maior valor de importação sendo produzidos por rota biotecnológica (NETO et al., 2011).

A expiração de patentes de diversos medicamentos biológicos é uma oportunidade para a criação de uma indústria biofarmacêutica no País. O mercado dos biossimilares ainda é pequeno, estimando-se um valor global de US\$ 3,7 bilhões mas, até 2020, doze produtos, com mercado de US\$ 76 bilhões terão suas patentes extintas. O investimento não parece ser um problema de grande magnitude para a indústria brasileira, pois as estimativas sobre os custos para a construção de uma planta de produção de medicamentos biológicos oscilam entre 30 e 200 milhões de reais (NETO et al., 2011).

Mas um desafio para essa produção é a alta complexidade do processo produtivo, em etapas como a identificação da nova molécula, desenvolvimento de um banco de células, definição da formulação farmacêutica pretendida, produção de escala-piloto, ensaios pré-clínicos e clínicos.

A indústria farmacêutica brasileira movimenta anualmente cerca de US\$ 10 bilhões, correspondendo ao $6^{0}$ maior mercado mundial da indústria. As empresas de capital brasileiro respondem por aproximadamente $20 \%$ do faturamento do setor e fazem produtos maduros ou com tecnologia transferida por parcerias internacionais mediante pagamento de royalties ou participação financeira. Por causa do foco em genéricos e da baixa produção farmoquímica, as atividades de $\mathrm{P} \& \mathrm{D}$ nas empresas de capital brasileiro ainda estão geralmente restritas à fase de formulação. Elas são pouco intensivas em P\&D, em relação ao padrão mundial do setor. (ANDRADE, 2015).

Assim, um gargalo importante refere-se à inexistência de empresas envolvidas em atividades de desenvolvimento e produção de medicamentos biológicos com escala suficiente para estruturar uma cadeia produtiva e gerar fluxos de conhecimento mais densos, inclusive integrando fornecedores e prestadores de serviços tecnológicos capazes de induzir processos coletivos de capacitação e diversificação. A escassez de serviços tecnológicos eleva o custo de desenvolvimento na etapa mais dispendiosa, a realização de ensaios clínicos. Também há escassez de recursos humanos capacitados em biotecnologia.

Entretanto, nos últimos anos, com a inclusão da biotecnologia como área prioritária da política industrial brasileira, o importante sistema acadêmico de ciência e tecnologia em saúde, ampliação dos fundos de financiamento e subvenção à inovação e das políticas de desenvolvimento produtivo do Ministério da Saúde, criaram-se condições favoráveis à capacitação tecnológica da indústria farmacêutica nacional. A expiração de patentes de produtos biológicos de alto custo para o SUS abre a oportunidade de produção local de "biossimilares", a exemplo do sucesso que as empresas farmacêuticas brasileiras obtiveram ao longo dos últimos anos com a fabricação de medicamentos genéricos. (ANDRADE, 2015).

Destaca-se a formação das Parcerias de Desenvolvimento Produtivo, que visam ampliar a capacidade de regulação dos preços de produtos adquiridos pelos SUS, internalizando a tecnologia de produção de fármacos nos laboratórios públicos através de processos de transferência de tecnologia e estimulando a produção local de produtos de alto custo e/ou grande impacto sanitário e social. As parcerias envolvem a associação de pelo menos um laboratório público com um laboratório privado, que é o responsável pelo desenvolvimento e transferência de tecnologia de produção. Entre 2009 e 2013, foram formalizadas 102 parcerias envolvendo a produção de medicamentos e equipamentos médicos, onze das quais referentes a produtos biológicos. (BRITTO E VARGAS, 2015).

Com isto, a participação das empresas de capital brasileiro vem aumentando, principalmente no segmento de genéricos e similares de síntese química. Elas vêm intensificando seus esforços inovativos, 
viabilizando estratégias de diversificação, crescimento e manutenção da rentabilidade (BRITTO E VARGAS, 2015).

A tabela 1 traz evidências sobre o panorama acima apresentado. Ela mostra que houve aumento substantivo do esforço em inovação entre as empresas farmacêuticas de capital brasileiro. Em um cenário de concentração de mercado, as empresas, em geral se tornaram mais intensivas em tecnologia, embora o número de empresas que fazem atividades de P\&D tenha declinado em todas as categorias, menos entre as empresas multinacionais maiores. Entre os indicadores de esforço tecnológico com resultado positivo, destacam-se o aumento da intensidade de gastos em $\mathrm{P} \& \mathrm{D}$, principalmente entre as empresas nacionais menores, o aumento generalizado da participação dos gastos em $\mathrm{P} \& \mathrm{D}$ em relação ao gasto inovativo total, indicando uma maior qualidade do gasto em inovação e o aumento do tamanho das equipes de P\&D.

Tabela 1 - Informações básicas sobre firmas inovadoras, esforços inovativos em cooperação e apoio governamental por porte e origem do capital - Setor Farmacêutico

\begin{tabular}{|c|c|c|c|c|c|c|c|c|}
\hline & \multicolumn{2}{|c|}{$\begin{array}{c}\text { Mais de } \\
500 \text { - Multi- } \\
\text { nacional }\end{array}$} & \multicolumn{2}{|c|}{$\begin{array}{c}\text { Mais de } \\
500 \text { - Na- } \\
\text { cional }\end{array}$} & \multicolumn{2}{|c|}{$\begin{array}{c}\text { Menos de } \\
500 \text { Multi- } \\
\text { nacional }\end{array}$} & \multicolumn{2}{|c|}{$\begin{array}{c}\text { Menos de } \\
500 \mathrm{Na}- \\
\text { cional }\end{array}$} \\
\hline & 2003 & 2011 & 2003 & 2011 & 2003 & 2011 & 2003 & 2011 \\
\hline Empresa Total & 21 & 23 & 20 & 30 & 38 & 44 & 543 & 361 \\
\hline Empresas inovadoras & 14 & 16 & 17 & 26 & 12 & 25 & 271 & 180 \\
\hline Porte (receita/empresa,milhões) & 458 & 815 & 256 & 497 & 47 & 66 & 5 & 12 \\
\hline Número firmas com P\&D & 13 & 11 & 17 & 25 & 3 & 4 & 99 & 87 \\
\hline Pessoal em P\&D & 106 & 369 & 347 & 1241 & 46 & 74 & 415 & 1414 \\
\hline Intensidade gastos em P\&D & 0,2 & 1,0 & 1,0 & 3,3 & 0,2 & 0,9 & 1,0 & 4,7 \\
\hline$\%$ gastos $\mathrm{P} \& \mathrm{D} /$ gastos inovativos & 6,2 & 36,5 & 25,6 & 58,0 & 12,9 & 49,6 & 26,3 & 49,5 \\
\hline $\begin{array}{l}\text { No de pessoas em P\&D por firma } \\
\text { com P\&D }\end{array}$ & 8 & 32 & 20 & 51 & 18 & 19 & 4 & 16 \\
\hline Pessoal em P\&D/pessoal total & 0,52 & 1,41 & 1,51 & 2,63 & 0,63 & 1,07 & 1,43 & 6,11 \\
\hline Taxa de cooperação inovadoras & 35,7 & 7,5 & 47 & 58,9 & 0,0 & 35,3 & 8,1 & 32,5 \\
\hline $\begin{array}{r}\% \text { de inovadoras - cooperação com } \\
\text { Universidades }\end{array}$ & 21,5 & 0 & 41,2 & 49,6 & 0,0 & 32,1 & 3,2 & 23,6 \\
\hline $\begin{array}{r}\% \text { de empresas inovadoras que } \\
\text { recebem apoio do governo }\end{array}$ & 7,2 & 36,3 & 35,3 & 100 & 0,0 & 0,0 & 16,5 & 46,7 \\
\hline
\end{tabular}

Fontes: tabulações especiais da Pintec (2003) e Pintec (2011)

O crescimento do esforço em inovação foi maior entre as empresas de capital brasileiro e, entre estas, as empresas menores, com duas exceções, "número de firmas com atividades de P\&D” e "percentual dos gastos em P\&D sobre gastos inovativos”.

Também se observa, na tabela 1, uma intensificação da cooperação tecnológica das empresas de capital brasileiro, em particular com as Universidades. Junto com o maior interesse das empresas em inovar, a política industrial do governo incorpora mecanismos de financiamento à relação Universidade/ empresa, o que também possivelmente explica este aumento.

Por fim, a tabela mostra o aumento do percentual de empresas com apoio do governo, que muito influiu na maior dinâmica inovativa das empresas de capital brasileiro. 


\section{A EXPERIÊNCIA DO LABORATÓRIO CRISTÁLIA NA ESFORÇOS CAPACITAÇÃO EM BIOTECNOLOGIA:}

\subsection{Perfil de atuação e desempenho}

O Laboratório Cristália é uma empresa de referência em inovação entre as indústrias farmacêuticas nacionais, sendo a maior produtora de matérias-primas dentre os laboratórios estabelecidos no país, com atuação no setor hospitalar, biológico, farmoquímico, genéricos, corporais e imunobiológico, contando com mais de 2.500 funcionários. A empresa foi criada em 1972 e seus segmentos de atuação são o hospitalar (anestésicos), que é o principal, disfunção erétil, AIDS, sistema nervoso central (distúrbio bipolar, esquizofrenia, depressão), dor e inflamação, dermatologia e estética. O Cristália é o maior produtor de medicamentos hospitalares na América Latina, estando presente em cerca de $95 \%$ dos hospitais brasileiros. Esta liderança foi um dos fatores responsáveis por possibilitar o crescimento da empresa e a consequente estratégia de diversificação para a biotecnologia.

Em 2013, o faturamento da empresa atingiu R \$ 1,4 bilhões, dividido por cinco Unidades de Negócio: 1) Hospitalar: comercializa anestésicos, adjuvantes e narcoanalgésicos; 2) Biológica: atua predominantemente em clínicas de radiologia, hemodiálise, cirurgia, oncologia e odontologia, além de comercializar escovas para a assepsia de cirurgiões; 3) Farma: dedicada aos produtos que são vendidos em farmácias e drogarias; 4) Genéricos: produtos para psiquiatria, neurologia, cardiologia, ginecologia, oftalmologia, dermatologia, dentre outros; 5) Corporis: produtos de dermatologia estética e dermocosméticos. Na sede empresa, em Itapira, SP, operam três plantas industriais: Farmoquímica, Biotecnologia e Farmacêutica. As duas primeiras são encarregadas pela produção de matérias primas, que alimentam a terceira, que faz a formulação farmacêutica e embalagem.

O Cristália também exporta produtos farmacêuticos terminados e matérias-primas para mais de 30 países da América Latina, Ásia, África e Oriente Médio. Os principais produtos exportados são dos segmentos de anestésicos e seus adjuvantes, analgésicos, produtos psiquiátricos e antirretrovirais. O laboratório tem a intenção estratégica de se expandir os Estados Unidos,, Europa e Japão, onde o atendimento a normas internacionais mais rigorosas é fator fundamental. Uma nova fábrica da empresa foi inaugurada em 2010, com padrões exigidos pelo US-FDA (Food and Drug Administration) e EMA (European Medicines Agency). Visando viabilizar a entrada nos mercados mais competitivos do mundo, o Laboratório Cristália obteve o reconhecimento da United States Pharmacopeia (USP), órgão norte-americano de influência mundial que fornece padrões a serem seguidos para o desenvolvimento de diversas substâncias

\subsection{Laboratórios e infra estrutura de P\&D}

A evolução da empresa evidencia um processo paulatino de construção, fortalecimento e diversificação de competências. A partir de 1983, a empresa consolidou suas atividades de P\&D, com a criação do Núcleo de Desenvolvimento Farmoquímico. A partir de 1988, o laboratório passou a fabricar insumos, para utilização nos medicamentos de seu portfólio e, posteriormente, para venda e exportação. A diversificação para a Farmoquímica foi crucial para o fortalecimento da capacidade inovativa inicial do Cristália, já que é muito mais difícil inovar na formulação (principalmente por questões regulatórias) do que na produção de matérias-primas.

Com a expansão para a farmoquímica, o Cristália procurou construir as capacidades técnicas necessárias para atividades de P\&D, correspondentes ao domínio das etapas de concepção do projeto, desenvolvimento do princípio ativo, estudos pré-clínicos e clínicos, farmacotécnica e, finalmente, produção e comercialização de medicamentos. No segmento farmacêutico, desde o início do desenvolvimento de um projeto de um novo produto, é preciso analisar como será classificado o medicamento (inovador, similar, biológico etc.) e que documentos serão apresentados à Anvisa, como parte da análise de viabilidade. Quando se tratam de produtos inovadores, as exigências são maiores, já que não há um medicamento de referência para análise de comparabilidade. Os ensaios clínicos são mais 
complexos, sendo necessária anuência prévia da Anvisa para sua realização, um processo moroso, na média com oito meses de espera.

O foco estratégico no desenvolvimento próprio de P\&D levou o Cristália, em 2009, a investir cerca de R\$ 30 milhões na construção de um Centro de Pesquisa, Desenvolvimento e Inovação (PD\&I), dotado de laboratórios e infraestrutura necessários para o desenvolvimento de seus projetos, e que passou a concentrar o planejamento e a coordenação das pesquisas, assim como atividades internas de P\&D. Atualmente, este centro é composto por quatro departamentos, cada um coordenando um conjunto de projetos: o de Biotecnologia e a Divisão Farmoquímica coordenam projetos para produção de princípios ativos; o Departamento de Novos Produtos gerencia projetos de desenvolvimento de genéricos e similares e dos medicamentos relacionados às Parcerias Público-Privadas e o laboratório de Pesquisa e Inovação (P\&I), que trabalha com a gestão dos projetos de inovação incremental (geralmente associadas às novas formulações ou métodos de liberação) e inovações radicais (geralmente novos princípios ativos, farmoquímicos ou biotecnológicos). Este laboratório também gerencia a interações com pesquisadores externos, como os das universidades e centros de pesquisa, privilegiando um modelo de parcerias com a academia. Os ensaios pré-clínicos sujeitos a maiores regulamentações são terceirizados para a Unifesp, Escola Paulista de Medicina, etc. As atividades de pesquisa articulam-se com gestão de patentes, para avaliar a liberdade de exploração (Freedom to Operate - FTO), verificando se as moléculas e as rotas de síntese (método de produção) não estão protegidas por patentes. Em 2013, os investimentos em P\&D da empresa atingiram $11 \%$ da receita líquida de vendas.

Inicialmente, o Cristália seguiu uma estratégia de inovação baseada na cópia de medicamentos existentes, a partir da engenharia reversa. A diferença é que ele considerou a cópia também como ponto de partida para estruturar o seu processo de inovação, a exemplo do que fizeram os países asiáticos. A empresa intensificou o lançamento de medicamentos exclusivos a partir da década de 1980, somando até hoje 27 produtos. Essa estratégia foi uma das bases da ampliação das competências técnicas no desenvolvimento de medicamentos, culminando com o lançamento de dois produtos novos para o mundo: Helleva, um medicamento para disfunção erétil, e Alimax, uma vacina desenvolvida a partir do antígeno da hepatite B administrada pela via oral. Em 2013, o Departamento de P\&D tinha um portfolio de 150 projetos, dos quais $60 \%$ podem ser considerados inovações radicais. Isso permite projetar perspectivas cada vez mais inovadoras para a empresa no futuro.

Refletindo essa trajetória, em 2007, a empresa recebeu o PRÊMIO FINEP de Inovação Tecnológica Nacional, concedido com base nas suas atividades de P\&D, inovação e geração de patentes.

\subsection{Qualificação do quadro de pessoal}

No caso das atividades de $P \& D$, a principal dificuldade é encontrar mão de obra qualificada. O período mínimo de treinamento para os profissionais atingirem um nível mínimo de mínimo de maturidade é de pelo menos um ano. Apesar do foco na contratação de Doutores (em Química, Biotecnologia e áreas afins), é difícil encontrar profissionais qualificados e a empresa inclusive busca profissionais no exterior. Em 2013, a empresa mobilizava 155 profissionais em atividades de P\&D, equivalentes a 7,5\% do quadro de pessoal. Do total de pessoal em P\&D, aproximadamente $20 \%$ era composto por pessoal com nível de pós-graduação. O recrutamento é 100\% internalizado, uma decisão estratégica para evitar vazamentos de informações confidenciais. Para viabilizar o processo de diversificação tecnológica, o laboratório contratou profissionais com alta capacidade técnica, identificados com a inovação. Os programas de treinamento são gerenciados pela área de RH, a partir de demandas repassadas pelas áreas operacionais. Cerca de $80 \%$ das demandas são para treinamentos técnicos, a maioria realizada internamente. As maiores exceções são das áreas técnicas, especialmente Biotecnologia. Os relacionamentos de confiança com a academia constituem um importante fator de apoio para suprir o gargalo de RH especializados para atividades de pesquisa. Mesmo assim, a falta de mão de obra qualificada em algumas áreas específicas tem obrigado a empresa a enviar alguns de seus pesquisadores para estudar no exterior. 


\subsection{Processo de pesquisa e desenvolvimento em biotecnologia}

O projeto para ingressar na Biotecnologia se iniciou em 2002, com a empresa incorporando, nos seus quadros, cientistas brasileiros que já tivessem experiência na área. Nesta etapa inicial, as plataformas tecnológicas para a produção de biofármacos privilegiaram microrganismos (bactérias ou leveduras, com destaque para o E. coli e células animais (CHO), com tecnologia relativamente conhecida. O foco em células animais foi importante porque os microrganismos são limitados em sua capacidade de expressão de moléculas (proteínas) mais complexas, como é o caso das proteínas humanas (e principalmente dos anticorpos monoclonais). O hormônio do crescimento humano foi o primeiro produto biotecnológico do Cristália. Entre 2002 e 2006, sua produção era feita em escala de bancada. Paulatinamente, a empresa internalizou as etapas de biologia molecular e engenharia genética que passaram a ser conduzidas nos laboratórios de PD\&I., visando a consecução de projetos em escala industrial, incluindo processos chaves de engenharia genética, fermentação, purificação e caracterização.

A expansão para a biotecnologia mais moderna foi importante na medida em que os anticorpos monoclonais apresentam um maior potencial terapêutico, podendo podem ser direcionados para o tratamento de doenças específicas, reduzindo os possíveis efeitos colaterais. No entanto, os desafios da ampliação da escala são maiores, pois esses produtos são muito mais complexos que o hormônio de crescimento, que por sua vez, é mais complexo do que uma molécula sintetizada quimicamente. Outra linha de desenvolvimento é a farmacogenômica, que trabalha a partir da constatação da individualidade da codificação genética de cada pessoa. Outra linha de ainda é o uso de microrganismos para produção de antibióticos (como a penicilina), que, de certa forma, revigora antigas técnicas de produção biológica. Por fim, existem tentativas de utilizar a nanotecnologia em conjunto com a biotecnologia (por exemplo, usar nanorobôs para direcionar o biofármaco dentro do organismo).

Com os avanços das pesquisas no campo biotecnológico, a empresa optou por um foco alinhado às demandas geradas pelo Ministério da Saúde, comunicadas através da lista de produtos estratégicos elaborada pela Secretaria de Ciência, Tecnologia e Insumos Estratégicos, buscando-se uma verticalização da produção de biofármacos, com o objetivo de diminuir a dependência do país em relação ao mercado externo para o fornecimento de insumos estratégicos. No final de 2007, a empresa constituiu uma equipe de biotecnologia que, desde então, vem desenvolvendo parcerias com universidades e institutos de pesquisa, a qual tem sido responsável por aproximadamente um quarto dos investimentos totais em PD\&I da empresa. A empresa também se beneficiou da disponibilidade de profissionais capacitados no mercado após a compra da Biobras por um laboratório multinacional. O projeto da planta de biotecnologia foi pensado para ampliação de escala, pois isso permite melhores análise de viabilidade técnica-econômica dos novos produtos. A planta piloto de microrganismos teve projeto iniciado em 2007, mas , com a crise de 2008, só entrou em funcionamento em 2012. A infraestrutura de P\&D em biotecnologia inclui laboratórios de modelagem molecular, biologia molecular, cultivo de células / fermentação, processos de purificação, pesquisa de novos produtos, ampliação de escala (intermediária, piloto ou industrial), desenvolvimento analítico e laboratório de testes (estabilidade, teor, bioequivalência, biodisponibilidade e afins). A biotecnologia pode também ser usada para reações em etapas intermediárias da produção de farmoquímicos.

A estratégia de entrada do Cristália direcionou-se para biossimilares com patentes em vias de expirar. Além das parcerias com universidades, a empresa estabeleceu uma parceria com a Alteogen (Coreana) para os desenvolvimento de anticorpos monoclonais, a partir do acúmulo de competências no desenvolvimento com o uso de células animais. O Cristália produz produtos biológicos, mas nenhum ainda está no mercado. O marco regulatório já está definido (biossimilares), mas o desafio da empresa é atender a todas as exigências. Existem diversas vias de registro de produtos biológicos, uma delas por comparabilidade, que é a via escolhida pela empresa, o que envolve caracterização físico-química, caracterização in vivo e ensaios clínicos nas fase I a III. A estrutura da planta industrial do Cristália apresenta uma capacidade produtiva que pode ser considerada piloto para alguns produtos e industrial 
para outros. Nesse sentido, além dos esforços de escalonamento na planta produtiva, torna-se importante a capacitação para elaborar protocolos para os ensaios clínicos.

Note-se que o acesso a fornecedores internacionais do biofármaco é problemático, pois esses tem sido gradativamente sendo adquiridos por companhias farmacêuticas globais, restringindo o fornecimento para o Cristália, que se encarregava apenas da formulação. Inicialmente, o Cristália adquiriu uma célula no exterior, adequando os processos à regulação mais recentes para sintetizar o biofármaco, mas essa célula é mantida por um Banco de Células dos EUA e para usá-las a empresa precisa pagar royalties ao detentor. Para contornar essa limitação, a empresa decidiu buscar na biodiversidade brasileira outras células (microrganismos) capazes de sintetizar o biofármaco, tendo que desenvolver uma metodologia de screening capaz de verificar se havia algum o microrganismo que produzisse a molécula alvo, se a quantidade produzida era viável e se seria possível isolá-lo de outros microrganismos. Após identificar um microrganismo que atendia a esses quesitos, foi necessário solicitar autorização de exploração comercial para o CGEN. A empresa registrou pedido de patente para o uso do microrganismo. Além do sucesso, a metodologia de screening permitiu identificar microrganismos ainda não caracterizados e seu processo de evolução.

5.5 Patentes

O Cristália detinha, em junho de 2016, 89 patentes concedidas, 32 das quais resultantes de parcerias com universidades.

Para viabilizar a implementação da estratégia inovativa da empresa, baseada em esforços de aperfeiçoamento de patentes já existentes, 74 patentes foram adquiridas até 2014. O departamento de patentes presta serviço para clientes internos (farmoquímica, biotecnologia e farmacêutica) e parceiros (universidades e centros de pesquisa). O Cristália preferiu internalizar a gestão dos registros de patentes. Para todos projetos de inovação, o departamento faz um pesquisa prévia de proteção patentária para determinar se á liberdade de exploração. O Cristália também apoia a obtenção de patentes por universidades através de contrato de licença.

\subsection{Relação com universidades num contexto de "inovação aberta"}

Para viabilizar sua estratégia inovativa, a empresa procura complementar suas atividades de P\&D interno com atividades de P\&D externo. Em 2013, os gastos com atividades de P\&D externo equivaliam a 5,4\% do valor da receita líquida de vendas e a $47 \%$ do valor dos dispêndios com P\&D interno. De todos os projetos que compõem hoje o portfólio de inovação do laboratório, inovações incrementais e radicais, 60\% tiveram origem externa. Se a universidade já possui a patente de um produto, a empresa a licencia. Mas se a universidade ou a empresa apresenta alguma proposta, a empresa torna-se co-autora do desenvolvimento do produto, com uma divisão do título e da exploração da patente gerada sendo repartida entre $50 \%$ para a empresa e $50 \%$ para a universidade. A importância que as universidades assumiram na geração de competências complementares em P\&D no Cristália indica um movimento na direção de um padrão baseado nos princípios da inovação aberta.

A articulação com universidade no caso do Cristália é anterior à estruturação de um arcabouço mais elaborado de incentivos governamentais, refletindo a busca de competências complementares capazes d impulsionar o processo de diversificação tecnológica. O relacionamento do Cristália com o setor acadêmico remonta à fundação da empresa, em 1972. As principais universidades brasileiras que a empresa mantém acordo são Unicamp, USP, Unifesp, UFRJ, UFRGS, UFMG, UFAM, UFSM e UECE, além de alguns centros pesquisa. A pesquisa realizada a partir do relacionamento do Cristália com as universidades caracteriza-se por ser aplicada, orientando-se para o desenvolvimento de produtos que posteriormente serão comercializados. A criação das principais leis de incentivos fiscais (Lei do Bem - lei $n^{0} .11 .196 / 2005$ e Lei da Inovação - lei $n^{0}$. 10.973/2004), estimulou a montagem de projetos mais bem elaborados, visando obter os incentivos definidos pela legislação. Além de investimento próprio em pesquisas de parceria, a empresa faz uso de editais e financiamentos de órgãos de fomento, mas identifica 
como entraves nesses financiamentos problemas relacionados ao compartilhamento da propriedade intelectual. O principal benefício identificado nesses projetos é o aprendizado mútuo, em termos de gestão de recursos e de projetos, mas também existem problemas relativos ao montante limitado dos financiamentos.

$\mathrm{Na}$ dinamização das atividades cooperativas com universidades, destaca-se o papel do Conselho Científico montado pelo Cristália, responsável pela seleção e capacitação de projetos externos, que funciona como uma espécie de consultoria em propriedade intelectual, mobilizando membros da academia, operando a partir de um acordo de confidencialidade entre os envolvidos. Esse Conselho, criado em 2004, constitui uma porta de entrada de projetos inovadores vindos das diversas universidades com as quais a empresa desenvolveu relacionamento. O Conselho Científico recebe continuamente projetos cujo enfoque deve ser uma das três áreas de atuação da empresa: Farmoquímica, Biotecnologia e Tecnologia Farmacêutica. A participação desses professores no Conselho não é vitalícia, podendo haver mudanças, quando se julgar necessário. No Conselho, não existe um contrato de trabalho, os professores recebem por assessoria dada aos projetos.

\subsection{Capacidade de absorção interna e limitações da estratégia}

O segredo do sucesso dessa iniciativa de diversificação do Cristália foi preparar-se para permitir a interação das universidades. O corpo técnico da empresa dedicado à inovação é em grande parte composto de mestres e doutores, o que facilita o diálogo e o entendimento mútuo. Além disso, a composição e a forma de operação do Conselho Científico, formado por profissionais multidisciplinares das áreas de química, biotecnologia, virologia, farmacologia, tecnologia farmacêutica, anestesia e pesquisa clínica, foi muito importante para a gestão do portfólio de inovação do Cristália. Desse modo, as parcerias com as diversas instituições de pesquisa, universitárias ou não, e com governos, agências de fomento ao desenvolvimento e profissionais da saúde convertem-se em canais de prospecção de novos projetos, avaliados através do Conselho Científico, seguindo um processo bem definido. O laboratório auxilia seus parceiros através do pagamento de bolsas de pesquisa, materiais, reagentes, equipamentos, treinamentos e infraestruturas dos laboratórios. Mais recentemente, além das parcerias com universidades, destacam-se a estruturação de PDPs com laboratórios oficiais a partir de 2010 (LAFEPE, Hemobras, Farmanguinhos, FURP, FUNED, Bahiafarma, Lab. Farm. da Marinha, Lifal, LQFEX-Exército, Iquego e LAFERGS).

\section{CONCLUSÕES}

A estratégia do Cristália para biotecnologia aponta para uma evolução progressiva da cópia para a inovação incremental e, finalmente, para a inovação radical, sendo este último o próximo passo almejado pela empresa. A empresa possui atualmente 55 pessoas ocupados na Biotecnologia, sendo um terço mestres e doutores. A demanda por pessoal qualificado na área deve aumentar quando os produtos forem registrados. A empresa está estruturando um projeto futuro para ensino técnico em Biotecnologia com o Senai. A experiência de diversificação tecnológica da empresa para o campo biotecnológico indica que a multidisciplinaridade requerida para o desenvolvimento de inovações é alta, o que confere muita complexidade ao seu desenvolvimento. O risco decorrente dessa complexidade pode muitas vezes inviabilizar um projeto, mas pode ser mitigado tanto através do acúmulo de conhecimento e do domínio das etapas envolvidas nesse desenvolvimento como ao se promover, antes do início de cada etapa do desenvolvimento, a participação dos membros da equipe, envolvidos diretamente nessa etapa, nas discussões técnicas e de planejamento. Nesse sentido, o Laboratório Cristália procurou construir as competências necessárias para viabilizar sua estratégia de inovação.

Estas questões são relevantes para a análise da inovação. Maior atenção deve ser dada às estratégias de RH e suas consequências. Em um contexto de inovação aberta, as estratégias de RH se tornam ainda mais 
relevantes. A inovação se torna cada vez mais sistêmica e o debate deve incorporar esta dimensão, principalmente em contextos de convergência tecnológica, onde o esforço de diversificação é maior porque a nova tecnologia genérica pode não ser relacionada à tecnologia usual da firma.

Por fim, cabe lembrar que todo este esforço para a inovação é apenas parte da construção de vantagens competitivas. Há outro conjunto de problemas para a introdução bem sucedida da nova tecnologia em uma empresa, que ocorrem à jusante do desenvolvimento tecnológico, nas etapas de produção e vendas. Por exemplo, as empresas eletrônicas estudadas por Gambardella e Torrisi (1998) ampliaram a diversificação tecnológica, mas não a de mercado, porque "... the downstream assets and capabilities that are needed to succeed in different markets have remained distinct.” (GAMBARDELLA; TORRISI, 1998, p. 446). Indústrias diferentes produzem bens com características diversas, em ambientes competitivos diferenciados. Este é certamente o caso da indústria farmacêutica, estudada neste trabalho, onde, por exemplo, frequentemente, a não posse de ativos específicos necessários à propaganda e à comercialização leva à absorção de empresas inovativas por grandes grupos econômicos do setor. O Laboratório Cristália se beneficia do fato de que a política industrial para a saúde inclui a dimensão das compras estatais. Outros casos podem não ter a mesma vantagem.

\section{BIBLIOGRAFIA}

ABDI, A. B. DE D. I. -. Estudo de Caso: Biotecnologia no Brasil: Sumário Executivo. Brasília: [s.n.]. Disponível em: <http://pi-tec-br.com/paginas/page1/Sumaario Executivo -Biotecnologia no Brasil.pdf $>$.

ANDRADE, P. A. Avaliação da Política Nacional de Ciência, Tecnologia e Inovação em Saúde: contribuições para a pesquisa \& desenvolvimento em biotecnologia em saúde (2004--2014). 2015.

BARNEY, J.; WRIGHT, P. On Becoming a Strategic Partner: The Role of Human Resources in gaining competitive Advantage. Cornell University. Center for Advanced Human Resource Studies. www. vocabulary. com/dictionary/talent, 1997.

BASOLE, R. C.; PARK, H.; BARNETT, B. C. Coopetition and convergence in the ICT ecosystem. Telecommunications Policy, p. 1-16, 2014.

BEUZEKOM, B. VAN; ARUNDEL, A. OECD biotechnology statistics-2006. 2006. BLOEM, J. et al. The Fourth Industrial Revolution. VINT research report, p. 1-39, 2014.

CHESBROUGH, H. Open Innovation: A New Paradigm for Understanding Industrial Innovation. Open innovation: researching a new paradigm, p. 1-12, 2006.

CANTNER, U.; VANNUCCINI, S. General Purpose Technologies as Emergent Properties, and the “Technological Multiplier” Hypothesis. 2014

CHESBROUGH, H.; BOGERS, M. Explicating Open Innovation: Clarifying an Emerging Paradigm for Understanding Innovation Keywords. New Frontiers in Open Innovation, p. 1-37, 2014.

DA SILVA TEIXEIRA, A. L. et al. Dimensões da capacidade de absorção, qualificação da mão de obra, P\&D e desempenho inovativo. Revista Brasileira de Inovação, v. 15, n. 1 jan/jun, p. 139-163, 2016.

DE OLIVEIRA MAZZONI, M. et al. A Participação da Engenharia nas Atividades de P\&D da Indústria de Transformação Brasileira: uma análise a partir da PINTEC. Revista Gestão \& Conexõeses, v. 2, n. 2, p. 102-122, 2013.

DRATH, R.; HORCH, A. Industrie 4.0: Hit or Hype? [Industry Forum]. Industrial Electronics Magazine, IEEE, v. 8, n. 2, p. 56-58, 2014.

FEDERAL MINISTRY OF EDUCATION AND RESEARCH. The new High-Tech Strategy Innovations for Germany. p. 1-58, 2014.

FONSECA, M. DA G. Documento setorial: biotecnologiaProjeto Perspectivas do Investimento no Brasil. Sistema Produtivo: Baseados em ciência. BNDES. UFRJ. Unicamp. Rio de Janeiro. Rio de Janeiro: [s.n.].

GAMBARDELLA, A.; TORRISI, S. Does technological convergence imply convergence in markets? Evidence from the electronics industry. Research policy, v. 27, n. 5, p. 445-463, 1998. 
GOLEMBIEWSKI, B.; SICK, N.; BRÖRING, S. The emerging research landscape on bioeconomy: What has been done so far and what is essential from a technology and innovation management perspective? Innovative Food Science \& Emerging Technologies, v. 29, p. 308-317, maio 2015.

HOLDREN, J. P. et al. Designing a Digital Future: Federally Funded Research and Development in Networking and Information Technology. p. 148, 2010.

KIM, N. et al. Dynamic patterns of industry convergence: Evidence from a large amount of unstructured data. Research Policy, v. 44, n. 9, p. 1734-1748, nov. 2015.

LAURSEN, K.; SALTER, A. Open for innovation: The role of openness in explaining innovation performance among U.K. manufacturing firms. Strategic Management Journal, v. 27, n. 2, p. 131-150, 2006.

NATIONAL SCIENCE FOUNDATION. Cyber-Physical Systems ( CPS ) PROGRAM SOLICITATION NSF 15-541 REPLACES DOCUMENT ( S ): p. 1-21, 2014.

NELSON, R. R.; WINTER, S. G. An evolutionary theory of economic change. [s.l.] Harvard University Press, 2009.

NETO, C. R. DE S. et al. Biotecnologia para saúde humana: tecnologias, aplicações e inserção na indústria farmacêutica. BNDES Setorial, n. 29, mar. 2009, p. 359-392, mar. 2009, 2009.

NETO, C. R. DE S.; LANDIM, A. B.; PIERONI, J. P. Lições da experiência internacional e propostas para incorporação da rota biotecnológica na indústria farmacêutica brasileira. BNDES Setorial, n. 34, set. 2011, p. 5-44, 2011.

PHARMA, E. World Preview 2013 Outlook to 2018. Returning to GrowthEvaluatePharma, , 2013.

PINTEC, I. Pesquisa de Inovação Tecnológica: 2003. Rio de Janeiro: [s.n.]. Disponível em: $<$ http://www.ibge.gov.br/home/estatistica/economia/industria/pintec/2003/default>.

PINTEC, I. Pesquisa de Inovação Tecnológica: 2008. Rio de Janeiro: IBGE-Departamento de indústria, 2010.

Disponível

em:

<http://www.ibge.gov.br/home/estatistica/economia/industria/pintec/2008/default>.

PINTEC, I. Pesquisa Industrial de Inovação Tecnológica: 2005IBGE--Instituto Brasileiro de Geografia e Estatística, Departamento de Indústria, Rio de Janeiro: IBGE. Rio de Janeiro: [s.n.].

Disponível

em: $<$ http://www.ibge.gov.br/home/estatistica/economia/industria/pintec/2005/default>.

PINTEC, I. Pesquisa Industrial de Inovação Tecnológica: 2011IBGE--Instituto Brasileiro de Geografia e Estatística, Departamento de Indústria, Rio de Janeiro: IBGE. Rio de Janeiro: [s.n.].

Disponível

em:

<http://www.ibge.gov.br/home/estatistica/economia/industria/pintec/2011/default>.

PRAHALAD, C. K.; RAMASWAMY, V. The new frontier of experience innovation. MIT Sloan management review, v. 44, n. 4, p. 12-18, 2003.

RISTUCCIA, C. A.; SOLOMOU, S. Can general purpose technology theory explain economic growth? Electrical power as a case study. European Review of Economic History, p. heu008, 2014.

ROSENBERG, N. Technological Change in the Machine Tool Industry, 1840-1910. The Journal of Economic History, v. 23, n. 04, p. 414-443, 1 dez. 1963.

SCHWAB, K. The fourth industrial revolution. Kindle Edition. Davos, Switzerland: World Economic Forum. Anais...2016

TEECE, D. J. et al. Dynamic capabilities and strategic management. Strategic management journal, v. 18, n. 7, p. 509-533, 1997.

WRIGHT, P. M.; DUNFORD, B. B.; SNELL, S. A. Human resources and the resource based view of the firm. Journal of management, v. 27, n. 6, p. 701-721, 2001.

ZANELLO, G. et al. The creation and diffusion of innovation in developing countries: A systematic literature review. Journal of Economic Surveys, 2015. 